\title{
Seleção de espécies e procedências de Pinus para região de Assis, Estado de São Paulo
}

\author{
Selection of Pinus species and provenances \\ for Assis region, State of São Paulo
}

\begin{abstract}
Francine Beatriz de Souza1, Miguel Luiz Menezes Freitas ${ }^{2}$, Mario Luiz Teixeira de Moraes ${ }^{3}$, Osmar Vilas Boas ${ }^{4}$ e Alexandre Magno Sebbenn ${ }^{5}$
\end{abstract}

\begin{abstract}
Resumo
O objetivo deste estudo foi à seleção de espécies e procedências de Pinus para a região de Assis, estado de São Paulo. O teste de espécie e procedências foi instalado na Floresta Estadual de Assis, no delineamento experimental blocos casualizados, com tratamentos representados por espécies (Pinus oocarpa, P. maximinoi e $P$. tecunumanii) e procedências por espécie, quatro blocos e parcelas quadradas com 49 plantas ( $7 \times 7$ ), no espaçamento de $3 \times 2,5 \mathrm{~m}$. Aos 21 anos após o plantio foram mensurados os caracteres: diâmetro à altura do peito (DAP), altura total (ALT), volume real individual (VOL) e a taxa de sobrevivência (SOBRE). Foram observadas diferenças significativas entre as espécies e procedências de diferentes espécies $(p<0.01)$ para o caractere SOBRE, indicando a possibilidade de seleção entre espécies e procedências. A divergência genética entre espécies variou entre os caracteres de crescimento de zero a 0,141 e foi de 0,683 para SOBRE, o que indica que a maior parte da variabilidade genética se encontra distribuída dentro das espécies para os caracteres de crescimento e entre espécies para a SOBRE. Foram observadas correlações genéticas e fenotípicas significativas e positivas entre os caracteres DAP e VOL (mínimo de 0,926; $p<0,01$ ) o que indica que a seleção para o DAP vai resultar em um aumento no VOL. Os resultados observados indicam que existem variações genéticas entre as espécies e procedências de diferentes espécies de Pinus para SOBRE e que a o $P$. oocarpa é mais indicada para o reflorestamento comercial nas condições ambientais de Assis.
\end{abstract}

Palavras-chave: Caracteres quantitativos; melhoramento genético; variação genética.

\begin{abstract}
The objective of the study was the selection of Pinus species and provenances for the region of Assis, state of São Paulo. The species and provenance test was established at the Assis State Forest, using the randomized blocks design with treatments represented by species (Pinus oocarpa, P. maximinoi and $P$. tecunumanii) and provenances of the species, four blocks and square plots with 49 plants $(7 \times 7)$, at a spacing of $3 \times 2.5 \mathrm{~m}$. At 21 years after planting the traits were measured: diameter at breast height (DAP), total height (ALT), individual real volume (VOL) and the survival rate (SOBRE). Significant differences were observed between species and provenances of different species $(p<0.01)$ for SOBRE, indicating the possibility of selection among species and provenances. The genetic divergence between species varied between growth traits from zero to 0.141 , and was 0.683 for SOBRE, indicating that most of the genetic variation is distributed within species for growth traits and between species for SOBRE. Genetic and phenotypic significant and positive correlations were observed between the DAP and VOL (minimum 0.926; $p<0.01$ ) indicating that selection for the DAP will result in an increase in the VOL. The results indicate that there are genetic variations among Pinus species and provenances of different species for SOBRE and $P$. oocarpa is most suitable for commercial reforestation in environmental conditions of Assis.
\end{abstract}

Keywords: Genetic variation; quantitative traits; tree breeding.

\footnotetext{
${ }^{1}$ Mestranda em Agronomia do Departamento de Fitotecnia. Faculdade de Engenharia de Ilha Solteira. UNESP - Universidade Estadual Paulista "Júlio de Mesquita Filho" / Faculdade de Engenharia de Ilha Solteira. Av. Brasil Centro, 56 - Caixa Postal 31 - 15385-000 - Ilha Solteira, SP, Brasil. E-mail: francinnysouza@yahoo.com.br.

${ }^{2}$ Doutor, Pesquisador Científico. IF -Instituto Florestal de São Paulo / Seção de Melhoramento Florestal - Rua do Horto, 931 - 02377-000 - Sao Paulo, SP, Brasil. CEP 02377-000. E-mail: miguellmfreitas@yahoo.com.br.

3Professor Titular do Departamento de Fitotecnia. Faculdade de Engenharia de Ilha Solteira. UNESP - Universidade Estadual Paulista "Júlio de Mesquita Filho" / Faculdade de Engenharia de Ilha Solteira. Av. Brasil Centro, 56 - Caixa Postal 31 - 15385-000 - Ilha Solteira, SP, Brasil. E-mail: teixeira@agr.feis.unesp.br.

${ }^{4}$ Doutor, Pesquisador Científico. IF -Instituto Florestal de São Paulo / Divisão de Florestas e Estações Experimentais, Seção de Floresta Estadual de Assis. Estrada Assis-Lutécia - Km 09 - Zona Rural - 19802-970 - Assis, SP, Brasil. E-mail: osmarvb@gmail.com.

${ }^{5}$ Doutor, Pesquisador Científico. IF -Instituto Florestal de São Paulo / Divisão de Florestas e Estações Experimentais, Estação Experimental de Tupi. Rodovia Luiz de Queiroz - Km 149,5 - 13400-970 - Piracicaba, SP, Brasil. E-mail: alexandresebbenn@yahoo.com.br.
} 
Souza et al. - Seleção de espécies e procedências de Pinus para região de assis, Estado de São Paulo

\section{INTRODUÇÃO}

O gênero Pinus engloba mais de 100 espécies com grande potencial a ser explorado em programas de melhoramento genético para diversos fins. Tais espécies são importantes fontes de matérias prima para vários segmentos industriais. Encontra-se nesse gênero algumas das espécies mais plantadas no mundo para a produção de madeira serrada, resina, papel e celulose (SHIMIZU; SEBBENN, 2008).

No Brasil são plantadas, com interesses comerciais, diversas espécies de gênero Pinus, devido ao seu crescimento, forma do fuste adequada e adaptação às condições edafoclimáticas do país. Em 2012, a área ocupada por plantios florestais de Eucalyptus e Pinus no Brasil totalizou 6.664.812 ha, sendo 23,4\% (1.562.782 ha) correspondentes aos plantios de Pinus (ABRAF, 2013).

Entre as espécies de Pinus plantadas no Brasil, destacam-se o P. oocarpa Schiede, P. maximinoi H. E. Moore e P. tecunumanii (Equilez \& Perry) Styles. O P. oocarpa é encontrado no México, Belize, Guatemala, Honduras e Nicarágua, em várias condições climáticas, com precipitação pluviométrica anual variando de 500 a $2.500 \mathrm{~mm}$ e altitude de 200 a $2.500 \mathrm{~m}$ (PERRY, 1991). A espécie apresenta melhor crescimento em solos bem drenados de encostas sob condições de clima temperado quente a subtropical. As árvores alcançam entre 20 a $30 \mathrm{~m}$ de altura e, em algumas situações até $35 \mathrm{~m}$, e diâmetro a altura do peito (DAP) entre 40 a $70 \mathrm{~cm}$ (AGUIAR et al., 2011). A madeira da espécie e útil para indústria de celulose, papel, construção civil e lenha, bem como apresenta potencial resinífero (KAGEYAMA et al., 1977; SHIMIZU; SEBBENN, 2008).

O P. maximinoi é a segunda espécie mais comum na América Central. Sua área de ocorrência natural estende-se desde o México, passando pela Guatemala, Honduras, El Salvador, ate o noroeste da Nicarágua (DVORAK et al., 2000). Ocorre em altitudes que variam entre 700 a $2.400 \mathrm{~m}$ e precipitação anual entre 900 e $2.200 \mathrm{~mm}$. Apresenta rápido crescimento, podendo chegar a 20 ou 40 $\mathrm{m}$ de altura e $1 \mathrm{~m}$ de DAP e sua madeira é leve, macia e de coloração clara, sendo muito resistente (SHIMIZU; SEBBENN, 2008). É adequada para uso na construção civil, celulose e painéis de fibras (WRIGHT; WESSELS, 1992). No Brasil, apresenta boa adaptação em regiões de clima subtropical e tropical. Esta espécie tem demonstrado alto incremento volumétrico e fuste de boa forma na região do cerrado, no sul do estado de São Paulo, em solo arenoso. Na região Sul, a cultura desta espécie é limitada devido à ocorrência de geadas severas (AGUIAR et al., 2011).

O P. tecunumanii é originada da América Central e América do Norte e adequada para reflorestamentos nas regiões tropicais brasileiras, por seu rápido crescimento, boa forma do fuste e baixa ocorrência de rabo-de-raposa (STYLES; HUGHES, 1988). A árvore pode atingir $50 \mathrm{~m}$ de altura e $120 \mathrm{~cm}$ de DAP, apresentando tronco é reto, geralmente livre de galhos até 20 a $30 \mathrm{~m}$ de altura e a madeira é de excelente qualidade (STYLES; HUGHES, 1988). Em relação à produtividade, entre as espécies de Pinus, o P. tecunumanii é um das que apresenta maior potencial, de acordo com as observações resultantes de uma série de ensaios internacionais (MOURA; VALE, 2002).

Um programa básico de melhoramento florestal pode ser basicamente dividido em três etapas (NAMKOONG, 1979): i) a seleção de espécies; ii) a seleção de procedências dentro da espécie mais adaptada e; iii) a seleção de progênies dentro das ou da procedência mais adaptada. O melhoramento genético florestal do gênero Pinus, tem-se voltado principalmente, para papel, celulose de fibra longa, madeira serrada e para extração de resina (SHIMIZU; SEBBENN, 2008). Uma das vantagens do melhoramento genético do Pinus é o aumento das taxas de ganho genético para o caráter produção volumétrica, produção de árvores com troncos mais retos, menor número de bifurcações e menor número de galhos grossos, melhorando o aproveitamento industrial da madeira.

O objetivo deste estudo foi a seleção de espécies e procedências de Pinus para a região de Assis, estado de São Paulo. Os objetivos específicos foram: i) determinar qual das espécies e procedências apresentam maior adaptação e crescimento para caracteres de crescimento e sobrevivência; ii) determinar como está distribuída a variação genética para os caracteres estudados entre e dentro de espécies e procedências; iii) determinar se existe correlações fenotípicas entre caracteres de crescimento. 


\section{MATERIAL E MÉTODOS}

\section{Local e delineamento experimental}

O teste de espécies e procedências foi instalado em novembro de 1991 na Floresta Estadual de Assis ( $22^{\circ} 35^{\prime} \mathrm{S}, 50^{\circ} 22^{\prime} \mathrm{O}$ e altitude média de $\left.562 \mathrm{~m}\right)$. Segundo a classificação de Koppen, o local da experimentação se encontra em uma zona de transição climática entre os tipos Cwa (clima temperado úmido com Inverno seco e Verão quente) e Cfa (clima temperado úmido com Verão quente). A precipitação média anual é de $1.400 \mathrm{~mm}$ e a temperatura média anual é de $21,8^{\circ} \mathrm{C}$. O solo do local foi caracterizado como Latossolo Vermelho Distrófico Alico Típico A, Moderado e textura média (EMBRAPA, 2006). O teste foi instalado no delineamento experimental blocos casualizados, com tratamentos representando espécies e procedências, quatro blocos e parcelas quadradas com 49 plantas ( $7 \times 7$ ), no espaçamento de $3 \times 2,5 \mathrm{~m}$ (Tabela 1 ). Uma bordadura dupla foi adicionada para reduzir o efeito ambiental das parcelas externas no experimento. As procedências representam um conjunto de sementes originadas de um numero desconhecido de arvores matrizes, visto tal informação ser desconhecida. Em julho de 2012 (21 anos após o plantio) foram mensurados os caracteres DAP, altura total (ALT), volume real individual (VOL) e a taxa de sobrevivência (SOBRE). O VOL foi estimado pela equação:

$$
V O L=\frac{1,57075(D A P / 100)^{2} A L T}{4},
$$

Tabela 1. Coordenadas geográficas, altitude, temperatura média e precipitação média anual de espécies e procedências de Pinus.

Table 1. Geographic coordinates, altitude, mean annual temperature and precipitation of Pinus species and provenances.

\begin{tabular}{|c|c|c|c|c|c|}
\hline Espécie/procedência & Latitude & Longitude & Altitude & $\begin{array}{l}\text { Temperatura } \\
\text { média anual }\end{array}$ & $\begin{array}{l}\text { Precipitação } \\
\text { média anual }\end{array}$ \\
\hline \multicolumn{6}{|l|}{ P. oocarpa } \\
\hline San Juan Ermita, Chiquimula & $14^{\circ} 33^{\prime} 32^{\prime \prime} \mathrm{N}$ & $89^{\circ} 20^{\prime} 31^{\prime \prime} \mathrm{W}$ & $1.160 \mathrm{~m}$ & $21,0^{\circ} \mathrm{C}$ & $1.742 \mathrm{~mm}$ \\
\hline San Luis Jilotepeque & $14^{\circ} 29^{\prime} 07^{\prime \prime} \mathrm{N}$ & $89^{\circ} 53^{\prime} 10^{\prime \prime} \mathrm{W}$ & $850-970 \mathrm{~m}$ & $22,3^{\circ} \mathrm{C}$ & $1.127 \mathrm{~mm}$ \\
\hline San Pedro Pinula & $14^{\circ} 39^{\prime} 44^{\prime \prime} \mathrm{N}$ & $89^{\circ} 50^{\prime} 47^{\prime \prime} \mathrm{W}$ & $1.015 \mathrm{~m}$ & $19,0^{\circ} \mathrm{C}$ & $1.900 \mathrm{~mm}$ \\
\hline \multicolumn{6}{|l|}{ P. maximinoi } \\
\hline San Raymundo & $14^{\circ} 43^{\prime} 00^{\prime \prime} \mathrm{N}$ & $90^{\circ} 39^{\prime} 00^{\prime \prime} \mathrm{W}$ & $1.585 \mathrm{~m}$ & $19,0^{\circ} \mathrm{C}$ & $1.333 \mathrm{~mm}$ \\
\hline Bosque Santa Rosita & $14^{\circ} 33^{\prime} 48^{\prime \prime} \mathrm{N}$ & $90^{\circ} 23^{\prime} 54^{\prime \prime} \mathrm{W}$ & $1.692 \mathrm{~m}$ & $20,0^{\circ} \mathrm{C}$ & $1.437 \mathrm{~mm}$ \\
\hline \multicolumn{6}{|c|}{ P. tecunumanii } \\
\hline Bosque San Geronimo, Salama, B.V. & $15^{\circ} 03^{\prime} 10^{\prime \prime} \mathrm{N}$ & $90^{\circ} 14^{\prime} 10^{\prime \prime} \mathrm{W}$ & $1.640 \mathrm{~m}$ & $21,5^{\circ} \mathrm{C}$ & $1.017 \mathrm{~mm}$ \\
\hline San Vicente, Salama, B.V. & $15^{\circ} 07^{\prime} 20^{\prime \prime} \mathrm{N}$ & $90^{\circ} 05^{\prime} 55^{\prime \prime} \mathrm{W}$ & $1.610 \mathrm{~m}$ & $19,5^{\circ} \mathrm{C}$ & $2.280 \mathrm{~mm}$ \\
\hline
\end{tabular}

\section{Análise de variância e estimativas dos componentes de variância}

As análises de variância foram realizadas utilizando-se o programa SAS (SAS, 1999). O teste F para os efeitos de blocos, espécies e procedências dentro de espécies foi calculado utilizando-se o procedimento GLM. As análises da variância para a estimativa dos componentes de variância foram conduzidas utilizando-se do método de REML (Restricted Maximun Likelihood) em combinação com o comando VARCOMP, devido ao desbalanceamento experimental, em termos do número desigual de procedências testadas por espécie e do número de árvores sobreviventes por parcelas. Para análise de variância e estimativa de componentes de variância, adotou-se o seguinte modelo:

$$
Y_{i j k l}=m+b_{i}+t_{j}+f_{j k}+(t b)_{i j}+(f b)_{i j k}+e_{i j k l}
$$

em que, $Y_{i j k l}$ é o valor fenotípico do $l$-ésimo indivíduo da $k$-ésima procedência da j-ésima espécie na $i$-ésima repetição (bloco); $m$ é o termo fixo da média geral; $b_{i}$ é o efeito fixo da $i$-ésima repetição; $t_{j}$ é o efeito fixo da j-ésima espécie; $f_{j k}$ é o efeito fixo da $k$-ésima procedência da j-ésima espécie; $(t b)_{j i}$ é o efeito da interação entre a j-ésima espécie e a $i$-ésima repetição; $(f b)_{i j k}$ é o efeito da interação entre a $k$-ésima procedência da j-ésima espécie na $i$-ésima repetição; $e_{i j k l}$ é o efeito da l-ésima árvore dentro da $k$-ésima procedência da $j$-ésima espécie na $i$-ésima repetição. Este último termo inclui os efeitos do erro dentro; $i=1 \ldots . b$ ( $b$ é o número de repetições, no caso blocos); $j=1 \ldots . t$ ( $t$ é o número de espécies, no caso 3$) ; k=1 \ldots f$ ( $f$ é o número de procedências dentro de espécies); $l=1 \ldots . n$ ( $n$ é o número de árvores por procedência). Da análise de variância foram decompostos os componentes 
de variância: $\phi_{\varepsilon}^{2}=$ variância genética entre espécies; $\phi_{p(\theta)}^{2}=$ variância genética entre procedências dentro de espécies; ${ }^{\sigma_{\hat{\theta}}^{*}}=$ variância da interação entre blocos e procedências dentro de espécies (variância ambiental).

\section{Estimativa de parâmetros genéticos}

Dos componentes de variância foi estimada a divergência genética entre espécies $\left(Q_{s t}\right)$, entre procedências dentro de espécies $\left(Q_{p}\right)$ e dentre procedências $\left(Q_{d}\right)$ :

$$
\begin{gathered}
Q_{s t}=\frac{\phi_{\varepsilon}^{2}}{\sigma_{d}^{2}+\phi_{p(\theta)}^{2}+\phi_{\varepsilon}^{2}} e \\
Q_{p}=\frac{\phi_{p(\theta)}^{2}}{\sigma_{d}^{2}+\phi_{p(\theta)}^{2}+\phi_{\varepsilon}^{2}}, e \\
Q_{d}=1-Q_{s t}-Q_{p} .
\end{gathered}
$$

\section{Correlações fenotípicas e genéticas entre caractere}

As correlações fenotípicas $\left({ }^{r_{F Z}}\right)$ e genéticas $\left({ }^{r_{g Z}}\right)$ entre as variáveis DAP, ALT e VOL foram estimadas dos valores individuais de acordo com as seguintes expressões:

$$
\begin{aligned}
& r_{F_{X Y}}=\frac{\sigma_{F_{X} F_{Y}}}{\sqrt{\sigma_{F_{X}}^{2} \sigma_{F_{Y}}^{2}}} e, \\
& r_{g_{X Y}}=\frac{\sigma_{p_{X} p_{Y}}}{\sqrt{\sigma_{p_{X}}^{2} \sigma_{p_{Y}}^{2}}}
\end{aligned}
$$

em que: $\sigma_{F_{X} F_{Y}} \mathrm{e}^{\sigma_{p_{X} p_{Y}}}$ são os produtos cruzados fenotípicos e genéticos das variáveis x e y, estimados das análises de covariância, $\sigma_{F_{x}}^{2}, \sigma_{p_{x}}^{2}, \sigma_{F_{y}}^{2}$ e $\sigma_{P y}^{2}$ são as variâncias fenotípicas e genéticas das variáveis x e y respectivamente (FALCONER, 1987).

\section{RESULTADOS E DISCUSSÃO}

\section{Variabilidade genética}

Foram observadas diferenças significativas $(\mathrm{p}<0,05)$ entre blocos pelo teste $\mathrm{F}$ da análise de variância para o caractere ALT, indicando que a instalação do teste no delineamento experimental de blocos ao acaso foi eficiente para controlar o ambiente (Tabela 2). Foram também detectadas diferenças significativas entre as espécies e procedências pelo teste $\mathrm{F}(\mathrm{P}<0,01)$ para SOBRE. Logo, existe a possibilidade de seleção entre espécies e procedências dentro de espécies. Em concordância, o teste de Tukey detectou diferenças significativas a 5\% de probabilidade entre as espécies para o caractere SOBRE, indicando que a média da SOBRE é significativamente maior no $P$. oocarpa do que para o P. maximinoi e P. tecunumanii (Tabela 3). Logo, o P. oocarpa foi é espécie mais indicada para o reflorestamento em Assis, visto a menor mortalidade. Contudo, não foram detectadas diferenças significativas entre as espécies e procedências para os caracteres de crescimento.

Tabela 2. Resultados do teste $\mathrm{F}$ da analise de variância para os quadrados médios.

Table 2. Results of $F$ test for variance analysis for mean squares.

\begin{tabular}{lccccc}
\hline Fonte de variação & GL & DAP & ALT & VOL & SOBRE \\
\hline Quadrado médio para bloco & 3 & 7,1052 & $41,3785^{\star *}$ & 0,0180 & 0,0034 \\
Quadrado médio para espécie & 2 & 0,6513 & 10,6129 & 0,0100 & $0,0818^{\star *}$ \\
Quadrado médio para procedência & 4 & 8,0304 & 2,2850 & 0,0171 & $0,0039^{\star *}$ \\
Coeficiente de variação experimental: $C V_{\rho}(\%)$ & - & 8,6 & 10,6 & 22,4 & 5,8 \\
\hline
\end{tabular}

** $\mathrm{P}<0,0 \mathrm{I} ; \mathrm{GL}=$ graus de liberdade; $\mathrm{DAP}=$ diâmetro à altura do peito; $\mathrm{ALT}=$ altura de plantas; $\mathrm{VOL}=$ volume individual; SOBRE $=$ sobrevivência. 
Tabela 3. Médias e teste de Tukey para caracteres em espécies e procedências dentro de espécies de Pinus.

Table 3. Means and Tukey test for traits in Pinus species and provenances within species.

\begin{tabular}{lcccc}
\hline Amostra & DAP $(\mathbf{c m})$ & ALT $(\mathbf{m})$ & VOL $\left(\mathbf{m}^{3} /\right.$ individuo $)$ & SOBRE $(\%)$ \\
\hline P. oocarpa & 26,13 & 20,32 & 0,5495 & $73,5^{\mathrm{a}}$ \\
P. maximinoi & 26,22 & 19,43 & 0,5238 & $47,0 \mathrm{~b}$ \\
P. tecunumanii & 25,70 & 18,22 & 0,4850 & $38,2 \mathrm{~b}$ \\
\hline P. oocarpa & & & & $65,0 \mathrm{abc}$ \\
San Pedro Pinula & 27,57 & 20,36 & 0,6138 & $74,5 \mathrm{ab}$ \\
San Luis Jilotepeque & 26,20 & 19,90 & 0,5397 & $81,0 \mathrm{a}$ \\
San Juan Ermita & 24,62 & 20,71 & 0,4951 & $48,5 \mathrm{bcd}$ \\
\hline P. maximinoi & & & & $45,5 \mathrm{~cd}$ \\
Bosque Santa Rosita & 25,15 & 18,87 & 0,4679 & $33,2 \mathrm{~d}$ \\
San Raymundo & 27,30 & 19,99 & 0,5796 & $43,2 \mathrm{~cd}$ \\
\hline P. tecunumanii & & & & 0,5278 \\
San Vicente, Salama & 26,52 & 19,20 & 0,4421 & \\
Bosque San Geronimo & 24,87 & 17,24 & & \\
\hline
\end{tabular}

Médias de SOBRE seguidas pelas mesmas letras não diferem entre si pelo teste de Tukey ao nível de $5 \%$ de significância. DAP = diâmetro á altura do peito; $A L T=$ altura de plantas; $\mathrm{VOL}=$ volume individual; SOBRE $=$ sobrevivência.

Comparando a média de SOBRE entre as procedências das diferentes espécies (Tabela 3), pelo teste de Tukey, não foram encontradas diferenças significativas entre as procedências da mesma espécie, apenas entre procedências de diferentes espécies ( $5 \%$ de probabilidade). Por exemplo, a procedência San Juan Ermita de P. oocarpa apresentou maior SOBRE dos que as procedências de P. maximininoi e P. tecunumanii e as procedências San Luis Jilotepeque e San Juan Ermita de P. oocarpa apresentaram maior SOBRE (mínimo de 74,5\%) dos que as procedências de $P$. tecunumanii (máximo de 43,2\%) e que a procedência San Raymundo de P. maximinoi. Estes resultados sugerem que existe a possibilidade de selecionar procedências de $P$. oocarpa e aumentar a produtividade de plantios na região de Assis.

Os caracteres DAP e SOBRE apresentaram coeficiente de variação experimental ( $\mathrm{CV}$ ) menores que $10 \%$, a ALT valor menor do que $20 \%$ e o VOL valor próximo a 20\% (Tabela 2). De acordo com a classificação de Garcia (1989) para o $C V_{\varepsilon}$ estimado em experimentos de campo, os caracteres DAP e SOBRE foram baixos $(<10 \%)$, para ALT foi médio $(<20 \%)$ e para o VOL foi próximo a médio $(22,4 \%)$. Baixos e médios valores de $C V_{\varepsilon}$ indicam precisão na estimativa de componentes de variância. Logo os componentes estimados para todos os caracteres foram confiáveis e apresentaram suficiente precisão experimental. O caractere VOL foi estimado pelo DAP e ALT, logo acumula os erros de mensuração de ambas as variáveis e maior valor de $C V_{\varepsilon}$ é esperado neste caso (TAMBARUSSI et al., 2010).

\section{Divergência genética entre espécies e procedências}

A divergência genética entre espécies $\left(Q_{s t}\right)$ variou entre os caracteres de zero (DAP e VOL) a 0,683 (SOBRE). Este resultado indica que a maior parte da variabilidade genética se encontra distribuída dentro das espécies, com excreção do caractere SOBRE, em que a maior parte da variabilidade genética esta entre espécies (Tabela 4). A divergência entre procedências dentro de espécies $\left(Q_{p}\right)$ variou entre os caracteres de 0,019 a 0,371 e foi menor que a divergência genética entre espécies para o DAP e VOL e dentro de procedências $\left(Q_{d}\right)$ para o DAP, ALT e VOL. Isso indica que as diferenças genéticas entre as procedências dentro das espécies para DAP e VOL são maiores do que as diferenças genéticas entre as espécies estudadas e a maior parte da variabilidade genética para os caracteres de crescimento ocorre dentro de procedências das diferentes espécies. Este resultado indica a possibilidade de se explorar esta variabilidade genética pela seleção massal dentro das procedências de diferentes espécies. Em outros termos, para os caracteres de crescimento, podem-se selecionar indivíduos superiores ou com maior crescimento dentro das procedências das três espécies $\mathrm{O}$ padrão observado de distribuição da variabilidade genética neste estudo, com maior variação entre indivíduos dentro de procedências para os caracteres de crescimento esta de acordo com os estudos realizados com Araucaria angustifolia (SEBBENN et al., 2003; 2004), Araucaria cunninghamii (SEBBENN et al., 2005), Cordia alliodora (SEBBENN et al., 2007), P. oocarpa (ETTORI et al., 2002) e Pinus patula ssp. tecunumanii (SEBBENN et al., 2005). 
Tabela 4. Divergência genética entre espécies, procedências dentro de espécies e dentro de procedências de Pinus. Table 4. Genetic divergence among Pine species, provenances within species and within provenances.

\begin{tabular}{lcccc}
\hline Fonte de variação & DAP & ALT & VOL & SOBRE \\
\hline Divergência genética entre espécies: $Q_{s t}$ & 0,000 & 0,141 & 0,000 & 0,683 \\
Divergência entre procedências dentro de espécies: $Q_{p}$ & 0,026 & 0,058 & 0,019 & 0,317 \\
Divergência genética dentro de procedências: $Q_{d}$ & 0,974 & 0,801 & 0,981 & - \\
\hline
\end{tabular}

$\mathrm{DAP}=$ diâmetro à altura do peito; $\mathrm{ALT}=$ altura de plantas; $\mathrm{VOL}=$ volume individual; SOBRE = sobrevivência.

\section{Correlações genéticas e fenotípicas entre os caracteres}

Foram observadas correlações genéticas $(0,955, \mathrm{p}<0,01)$ e fenotípicas $(0,926, \mathrm{p}<0,01)$ positivas e significativas entre os caracteres DAPxVOL. Isso demonstra que existe a possibilidade de se selecionar indivíduos das três espécies com base em observações fenotípica no DAP e alterar indiretamente a média genética e fenotípica do VOL e obter progressos em programas de melhoramento genético. Estes resultados eram esperados, devido a outros estudos com espécies arbóreas terem mostrado forte correlação entre tais caracteres (MISSIO et al., 2004; MOURA; VALE, 2002; SEBBENN et al., 2004; 2008; FREITAS et al., 2008). Este resultado e também obvio, visto que o VOL e estimado do DAP. A seleção quando é feita em variáveis com correlações significantemente positivas resulta em ganhos genéticos indiretos, ou seja, a seleção realizada em um caractere resulta em ganho indireto em outro caractere correlacionado (SAMPAIO et al., 2000).

\section{CONCLUSÕES}

Existem variações genéticas entre as três espécies de Pinus para sobrevivência;

Com base na sobrevivência, a espécie $P$. oocarpa e a mais indicada para reflorestamento comercial nas condições ambientais de Assis;

A maior parte da variabilidade genética para os caracteres de crescimento e adaptação se encontra distribuída dentro de espécies.

\section{AGRADECIMENTOS}

A realização deste estudo foi possível graças à concessão de bolsa FAPESP à autora Francine Beatriz de Souza (processo: 2013/02826-3). Os autores são gratos ao Prof. Dr. Alan Rodrigo Panosso (Departamento de Matemática da Faculdade de Engenharia de Ilha Solteira - UNESP) e a Prof ${ }^{\mathrm{a}}$. Dr ${ }^{\mathrm{a}}$. Daniela Sílvia de Oliveira Canuto (Departamento de Fitotecnia, Tecnologia de Alimentos e Sócio Economia da Faculdade de Engenharia de Ilha Solteira - UNESP), pela prévia revisão do Artigo.

\section{REFERENCIAS BIBLIOGRÁFICAS}

ABRAF. ASSOCIAÇÃO BRASILEIRA DE PRODUTORES DE FLORESTAS PLANTADAS. Anuário estatístico da ABRAF 2013: Ano base 2012. Brasília, 2013. 148 p. Disponível em: . Acesso em: 03 dez. 2014.

AGUiAR, A. V.; SOUSA, V. A.; FRITZSONS, E.; PINTO JR., J. E. Programa de melhoramento de Pinus da Embrapa Florestas. Colombo: Embrapa Florestas, 2011. 81 p. (Documentos, 233)

DVORAK, W. S.; GUTIÉRREZ, E. A.; GAPARE, W. J.; HODGE, G. R.; OSORIO. L. F; BESER, C.; KIKUTI, P. Pinus maximinoi. In: Conservation \& testing of tropical \& subtropical forest tree species by the Camcore Cooperative. Raleigh: College of Natural Resources, 2000. p. 106-128.

EMBRAPA - EMPRESA BRASILEIRA DE PESQUISA AGROPECUÁRIA. Sistema brasileiro de classificação de solos. 2.ed. Rio de Janeiro, 2006. 306 p.

ETTORI, L. C.; SEBBENN, A. M.; SATO, A.; MORAIS, E. Teste de procedências de Pinus oocarpa Schiede em três locais do Estado de São Paulo. Revista do Instituto Florestal, São Paulo, v. 14, n. 1, p. 39-51, 2002.

FALCONER, D. S. Introdução a genética quantitativa. Viçosa: UFV, 1987. 279 p. 
FREITAS M. L. M.; SEBBENN, A. M.; ZANATTO, A. C. S.; MORAES, E.; MORAES M. A. Variação Genética para caracteres quantitativos em população de Gallesia integrifolia (Spreng). Harms. Revista do Instituto Florestal, São Paulo, v. 20, n. 2, p. 165-173, 2008.

GARCIA, C. H. Tabelas para classificação do coeficiente de variação. Piracicaba: IPEF, 1989. 12 p. (Circular técnica, 171).

KAGEYAMA, P. Y.; VENCOVSKY, R.; FERREIRA, M.; NICOLIELO, N. Variação genética entre procedências de Pinus oocarpa Schiede na região de Agudos-SP. IPEF, Piracicaba, n. 14, p. 77-120, 1977.

MISSIO, R. F.; CAMBUIM, J.; MORAES, M. L. T.; PAULA, R. C. Seleção simultânea de caracteres em progênies de Pinus caribaea Morelet var. bahamensis. Scientia Forestalis, Piracicaba, n. 66, p. 161-166, 2004.

MOURA, V. P. G.; VALE, A. T. Variabilidade genética na densidade básica da madeira de Pinus tecunumanii procedente do México e da América Central no cerrado. Scientia Forestalis, Piracicaba, n. 62, p. 104-113, 2002.

NAMKOONG, G. Introduction to quantitative genetics in forestry. Washington: Forest Service, 1979,342 p. (Technical Bulletin, 1588)

PERRY, J. P. The Pines of Mexico and Central America. Portland: Timber, 1991. 231 p.

SAMPAIO, P. T. B.; RESENDE, M. D. V.; ARAÚJO, A. J. Estimativas de parâmetros genéticos e métodos de seleção para o melhoramento genético de Pinus caribaea var. hondurensis. Pesquisa Agropecuária Brasileira, Brasília, v. 35, n. 11, p. 2243-2253, 2000.

SAS INSTITUTE INC. SAS Procedures Guide. Version 8 (TSMO). Cary, 1999.

SEBBENN, A. M.; BOSHIER, D.; FREITAS, M. L. M.; ZANATTO, A. C. S.; SATO, A. S.; ETTORI, L. C.; MORAES, E. Results of an international, provenance trial of Cordia alliodora in São Paulo, Brazil at five and 23 years of age. Silvae Genetica, Frankfurt, v. 56, n. 3-4, p. 110-117, 2007.

SEBBENN, A. M.; PONTINHA, A. A. S.; FREITAS, S. A.; FREITAS, J. A. Variação genética em cinco procedências de Araucaria angustifolia (Bert.) O. Ktze. No Sul do estado de São Paulo. Revista do Instituto Florestal, São Paulo, v. 16, n. 2, p. 91-99, 2004.

SEBBENN, A. M.; PONTINHA, A. A. S.; GIANOTTI, E.; KAGEYAMA, P. Y. Genetic variation in provenance progeny test of Araucaria angustifolia (Bert.) O. Ktze. in São Paulo, Brazil. Silvae Genetica, Frankfurt, v. 52, n. 5-6, p. 181-184, 2003.

SEBBENN, A. M.; FREITAS, M. L. M.; MORAIS, E.; ZANATTO, A. C. S. Variação genética em procedências e progênies de Pinus patula ssp. tecunumanii no noroeste do Estado de São Paulo. Revista do Instituto Florestal, São Paulo, v. 17, n. 1, p. 1-15, 2005.

SEBBENN, A. M; VILAS BOAS, O.; MAX, J. C. M. Variação genética, herdabilidades e ganhos na seleção para caracteres de crescimento em teste de progênies de Pinus caribaea var. bahamensis aos 20 de idade em Assis-SP. Revista do Instituto Florestal, São Paulo, v. 20, n. 2, p. 103-115, 2008.

SHIMIZU, J. Y.; SEBBENN, A. M. Espécies de Pinus na silvicultura brasileira. In: SHIMIZU, J. Y. (Ed.). Pinus na silvicultura brasileira. Colombo: Embrapa Florestas, 2008. p. 49-74.

STYLES, B. T.; HUGHES, C. E. Variabilidad de los Pinus centroamericanos: taxonomia y nomenclatura de los Pinus y otras Gimnospermas. Honduras: CENIFA, 1988. 20 p.

Sci. For., Piracicaba, v. 44, n. 111, p. 675-682, set. 2016 DOI: dx.doi.org/10.18671/scifor.v44n111.13 
Souza et al. - Seleção de espécies e procedências de Pinus para região de assis, Estado de São Paulo

TAMBARUSSI, E. V.; SEBBENN, A. M.; MORAES, M. L. T.; ZIMBACK, L.; PALOMINO, E. C.; MORI, E. S. Estimative of genetic parameters in progeny test of Pinus caribaea Morelet var. hondurensis Barret \& Golfari by quantitative traits and microsatellite markers. Bragantia, Campinas, v. 69, p. 39-47, 2010.

WRIGHT, J. A.; WESSELS, A. Laboratory scale pulping of Pinus pseudostrobus, Pinus maximinoi and Pinus patula. IPEF International, Piracicaba, n. 2, p. 39-44, 1992.

Recebido em 28/01/2015

Aceito para publicação em 01/02/2016 